Escuela de Ciencias Sociales y Humanidades, UNED, Costa Rica https://revistas.uned.ac.cr/index.php/espiga ISSN: 1409-4002・e-ISSN: 2215-454X

\title{
Revista Espiga en la memoria histórica de la ECSH y de la UNED
}

\section{Revista Espiga in the historical memory of the ECSH and the UNED}

\section{La Revue Espiga dans la mémoire historique de I'ECSH et de I'UNED}

Pedro J. Ramírez Acosta *

Recibido: 2 de mayo de 2021 - Aceptado: 16 de junio de 2021

* Doctor en Educación, Universidad Estatal a Distancia (UNED), de Costa Rica. Msc. en Administración Pública, Universidad de Costa Rica (UCR). Licenciado en Teología y Filosofía de la Universidad Gregoriana y de la Universidad de Santo Tomás, respectivamente, Roma, Italia. Catedrático de la UNED y de la UCR. Exdirector de la Revista Espiga del 2000 al 2009. Este artículo se publica en conmemoración de los 20 años de la revista. Correo: pra413@gmail.com 


\section{Resumen}

El presente trabajo se propone rememorar el origen y propósito de la creación de Revista Espiga, en el contexto histórico y académico de la ECSH y de la UNED. Pone énfasis en la importancia y necesidad de una revista seria y científica, que ayudara a impulsar la investigación crítica y científica y la difusión de sus resultados. Expone, asimismo, parte de sus logros y problemas; $y$ al final se señalan algunos desafíos por espigar en la nueva sociedad digital.

Palabras clave: Antecedentes, investigación, desafíos.

\section{Abstract}

This paper aims to recall the origin and purpose of the creation of Revista Espiga, in the historical and academic context of the ECSH and the UNED. It emphasizes the importance and need for a serious and scientific journal, which would help to promote critical and scientific research and the dissemination of its results. It also exposes part of its achievements and problems; and at the end some challenges are pointed out to be gleaned for the new digital society.

Keywords: Background, challenges, research.

\section{Résumé}

Cet article vise à évoquer l'origine et le but de la création de la Revue Espiga dans le contexte historique et académique de l'École des Sciences Sociales et Humanités (ECSH) et de l'UNED. L'article met l'accent sur l'importance et le besoin d'une revue scientifique afin d'appuyer la recherche scientifique et critique ainsi que la diffusion de ses résultats. En outre, il expose ses réussites et ses contraintes. Finalement, il signale quelques défis auxquels la revue doit faire face dans la nouvelle société numérique.

Mots-clés: Antécédents, défis, recherche. 


\section{Introducción}

Escribir sobre el origen de Revista Espiga y su proyección en estos 22 años de existencia no es nada simple y pasajero. Aquí tiene mucho sentido aquella frase tantas veces repetida por los existencialistas franceses y alemanes de la década de 1960: «Existir es más que vivir». Espiga no solamente ha tenido vida, sino que también ha existido en la mente y en el quehacer cotidiano de la Escuela de Ciencias Sociales y Humanidades (ECSH) y sus pre-ocupaciones, pensamientos y publicaciones han trascendido a la Universidad Estatal a Distancia (UNED), al ámbito universitario costarricense, y a la sociedad.

En este sentido, me complace mucho escribir esta breve reseña histórica de Espiga a petición del Comité Editorial de la Revista, exponiendo los antecedentes y la importancia de su nacimiento, su atinado nombre escogido, sus alcances, sus principales problemas y los nuevos surcos para espigar en el futuro.

\section{Antecedentes históricos}

Revista Espiga fue creada en sesión número 1394-99, art. IV, el 23 de junio de 1999, por el Consejo Universitario, a solicitud de un grupo de profesores de la ECSH. Fue la primera revista académica de la UNED, surgida del propio claustro de la academia ¿Por qué la primera? Existían dos revistas anteriores: una, la Revista Nacional de Cultura, dirigida y editada por el Consejo de la Editorial Euned, y la segunda, Revista Innovaciones Educativas, dirigida por el Vicerrector Académico, cuya periodicidad era espontánea y sin vinculación expresa con las cuatro escuelas de la universidad, creadas diez años atrás, en sesión número 790-89, del 8 de febrero de 1989, por el Consejo Universitario de la UNED.

Algunos otros hechos explican este tardío nacimiento. La UNED fue creada en febrero de 1977, por ley de la República número 6044. Su modelo original estuvo centrado en lo que se llamó «entrega de la docencia» y en la producción de materiales, cuyo producto principal era la Unidad Didáctica. Este modelo estuvo inspirado en los europeos y norteamericanos de educación a distancia, que surgieron como respuesta a las demandas de educación de la Primera Revolución Industrial, a fines del siglo XVIII. Estos modelos operativos fabriles eran horizontales sistémicos, de input-output, semejantes a las cadenas de mando de las grandes fábricas industriales ${ }^{1}$.

En el caso de la UNED, al igual que otras famosas universidades, como la Open University de Inglaterra, la Fernuniversität de Alemania, la Universidad Abierta de Venezuela y la UNED de España, entre otras, evolucionó hacia un modelo más híbrido, es decir, de una Dirección de Centros Académicos con dos dependencias administrativas: a) la Oficina de ciencias del hombre y b) la Oficina de Ciencias Exactas y Naturales; estas Oficinas administraban grandes

1 Pedro Ramírez Acosta, «Elementos para el estudio de viabilidad de un Sistema de Educación a Distancia para la Universidad Autónoma de Nicaragua» (tesis de Maestría Académica, Universidad de Costa Rica, 1984), 153-158. 
áreas del conocimiento concentradas en programas docentes (o las llamadas carreras en la universidad tradicional), a una Dirección de Docencia ${ }^{2}$, creada en febrero de 1989, e integrada por cuatro escuelas: Ciencias Sociales y Humanidades, Educación, Administración y Ciencias Exactas y Naturales ${ }^{3}$. Este cambio en el modelo, de menos industrial y administrativo a más académico, se proponía fortalecer la academia con especialidades, grados y posgrados 4 .

¿Qué significaba fortalecer la academia y el espíritu académico en la UNED? Significó generar una transformación desde dentro, desde la esencia misma de la academia. No bastaba con cambiar ciertas estructuras administrativas, hacer nuevos materiales didácticos o mejorar la mal concebida «entrega de la docencia». Significaba romper las barreras de la reforma napoleónica. En Alemania, Humbolt inició, en el siglo XIX, una transformación de la educación superior, centrándola en la investigación, después reafirmada por teóricos modernos y actuales, que hicieron de la universidad una institución creadora de conocimientos 5 .

Con estos cambios se depuró el concepto de la democratización de la educación superior, uno de los objetivos principales de la educación superior a distancia. Democratizar la educación superior no consistía ni bastaba con la masificación de la universidad, no consistía en entregar títulos devaluados sin calidad. La sociedad, las empresas, los nuevos empleadores demandaban, ahora, individuos emprendedores con formación crítica, investigadores de su realidad económica, social y cultural, capaces de enfrentar y dar respuesta a los problemas urgentes para el logro de un desarrollo humano integral. El «medio maestro», como se le llamaba a la unidad didáctica, era insuficiente.

A finales de la década de 1990 la consigna y meta de la sociedad post-industrial y del conocimiento era un desarrollo humano integral. Las mejores universidades del mundo apostaban por un crecimiento basado en la calidad de la educación, o lo que Barrow ${ }^{6}$ llama «excelencia selectiva» y Tünnermann «excelencia académica»7.

Como era de esperar, estos nuevos aires de cambio llegaron a nuestras universidades en América Latina. En el caso de la UNED de Costa Rica, estas ideas estuvieron presente en el I Congreso Universitario (1989), que recomendó crear la Dirección de Docencia y las cuatro escuelas académicas. Entre las funciones principales de estas nuevas escuelas destacamos dos por su interés en la calidad académica:

- Velar por el nivel académico de los cursos (...)

\footnotetext{
2 UNED, Acuerdos sobre la creación de la Dirección de Docencia, edición provisional, 1990.

${ }^{3}$ Lester Osorno, Xinia Zúñiga Muñoz y Pedro Ramírez Acosta, «La Escuela de Ciencias Sociales y Humanidades en el treinta aniversario de la UNED», Revista Espiga 8, n. 18 (2009): 61-63, Doi: https://doi.org/ 10.22458/re.v8i18.1044.

${ }^{4}$ Rodrigo Barrantes Echavarría, Educación a Distancia (San José: EUNED,1998), 86-90.

${ }^{5}$ Unesco, «Declaración Mundial sobre la Educación Superior». DIÁLOGO, México OPI-LAC-Unesco, 1998.

${ }^{6}$ Clyde W. Barrow, «La estrategia de la excelencia selectiva», Perfiles educativos 19, n. ${ }^{\circ} 77$ (1997): 76-77.

7 Carlos Tünnermann, La Educación Superior frente al cambio (San José: EDUCA/CSUCA, 1997).
} 
- Estimular la participación de los profesores en propuestas de investigación, en coordinación con la Dirección de Investigación Institucional. ${ }^{8}$

Sin lugar a duda, en el I Congreso hubo un interés por un cambio hacia la calidad y hacia la investigación. Sin embargo, ese cambio, relacionado con la participación de los profesores en proyectos de investigación, tuvo fuertes obstáculos: Uno era la limitación y centralización del presupuesto de las escuelas, y, en segundo lugar, el viejo modelo de la Dirección de Investigación Institucional, cuyo presupuesto estaba destinado a investigaciones relacionadas con las unidades didácticas y a otras de interés institucional. En consecuencia, la investigación, por parte de profesores en áreas de su especialidad o de interés para el desarrollo de sus cursos, quedó paralizada en espera de nuevas oportunidades y cambios en la institución.

Otro hecho significativo en relación con las nuevas demandas de la sociedad post-industrial y de los países en vías de desarrollo, fue la creación de posgrados: especialidades, maestrías profesionales y académicas, y doctorados profesionales y académicos. La UNED creó su propio Sistema de Posgrado (SEP); en sesión número 1191-96, del 21 de febrero de 1996, se aprobó su reglamento. Por iniciativa del entonces director del SEP, consciente de las demandas señaladas arriba, los posgrados de la UNED nacieron bajo la inspiración del modelo de Burton Clark $(1983,1998)$, exrector de la Universidad de Berkeley, Estados Unidos. Este modelo contenía tres características esenciales: a) Planificación del proyecto, b) Centralidad de la investigación para producir conocimientos, innovación y competitividad y c) Perfil de formación profesional de los candidatos seleccionados, orientado a solucionar problemas ${ }^{9}$.

En consecuencia, hubo en la creación de los posgrados el interés por la centralidad de la investigación y por la selección de los candidatos. Por reglamento y para su funcionamiento, los posgrados eran planeados y propuestos por las escuelas y aprobados por el Consejo Universitario. Nacían y eran de las escuelas. Por su carácter sistémico era de esperar una intercomunicación de doble vía entre escuelas y posgrados. Ruidos estructurales en el sistema, como dicen los comunicadores, se interpusieron para impedir o retardar la intercomunicación.

¿Qué relación tienen todos estos hechos o antecedentes históricos con Revista Espiga? Hay una constante en todos ellos: necesidad de calidad, investigación, creación de nuevos conocimientos y publicación de los nuevos conocimientos. El ranking de las mejores universidades del mundo se mide por la cantidad y calidad de sus investigaciones científicas, tecnológicas y humanísticas publicadas. Estos nuevos conocimientos publicados se exponen en conferencias, congresos y simposios internacionales. Por tanto, era una necesidad latente la creación de una revista seria, científica y humanista en la ECSH.

\footnotetext{
8 Barrantes Echavarría, La Educación a Distancia..., 90-92.

9 Burton Clark, The Higher Education System: Academic Organization in Cross National-Perspectives (Berkeley: University of California Press, 1983).
} 


\section{Nacimiento de Revista Espiga}

Tres circunstancias propiciaron el nacimiento y desarrollo de Revista Espiga. La primera fue el retorno a la ECSH del exdirector de Posgrado en 1999, después de una intensa labor en la creación de las bases organizativas del SEP (reglamentos, normas y procedimientos) y puesta en marcha de los primeros 8 posgrados de las escuelas de la UNED.

En segundo lugar, fue determinante para la aprobación de la Revista el apoyo del vicerrector ejecutivo y el cambio de rector, en 1999. Si bien persistía el enfoque administrativo vertical centralizado, el cambio propició una nueva sensibilidad hacia lo académico, mayor comunicación y apertura con las escuelas y a las demandas de la sociedad.

Una tercera circunstancia, igualmente importante, fue la iniciativa coincidente de un grupo de profesores por la creación de una revista seria y con rigor académico, que fomentara la investigación, la innovación y el mejoramiento académico de programas, cursos, materiales didácticos y demás actividades de la ECSH. Ese grupo lo conformaba el coordinador del Programa de Estudios Generales, el director de la ECSH y otros distinguidos profesores encargados de cátedras y de cursos.

Se escribió la propuesta, que fue avalada por el Consejo de Escuela de ese período y se elevó al Consejo Universitario. Como se indicó arriba, la creación de Revista Espiga fue aprobada por dicho Consejo el 23 de junio, 1999. Sus principales características, que justificaron su aprobación eran: naturaleza humanista y crítica, abierta al análisis de los grandes y graves problemas de la sociedad actual y a las nuevas corrientes de pensamiento, promotora de la investigación por parte de los profesores de los diferentes programas o carreras de la Escuela y un medio de difusión de los mejores trabajos y avances de investigación de esta Unidad Académica, de otras escuelas y de investigadores externos de reconocido prestigio. Así, el sueño de una revista, que fuera la voz oficial de la Escuela, comenzó a dar sus primeros pasos.

\section{¿Por qué el nombre de Espiga y cuáles fueron sus raíces culturales y alcances?}

Ese equipo de profesores, antes mencionado, propuso varios nombres y entre ellos el de Espiga. Se pensó, también, en el nombre de Revista de Ciencias Sociales, pero ya existía una con ese mismo nombre en la Universidad de Costa Rica. Entonces, se acogió el de Espiga porque integraba el esfuerzo original humanista de la UNED: Una de las primeras Oficinas fue la de Ciencias del hombre, en la que estaba incluida el área de Ciencias Sociales y Humanidades ${ }^{10}$.

El exdirector del SEP y nuevo director de Revista Espiga había estudiado Filosofía y Teología en Europa, por lo que conocía el significado de espiga en la literatura bíblica y en la cultura de la media luna fértil, o sea, la de las naciones que bordeaban el Mediterráneo desde hace más de 10000 años. 


\section{Figura 1}

Diferentes portadas de la Revista Espiga

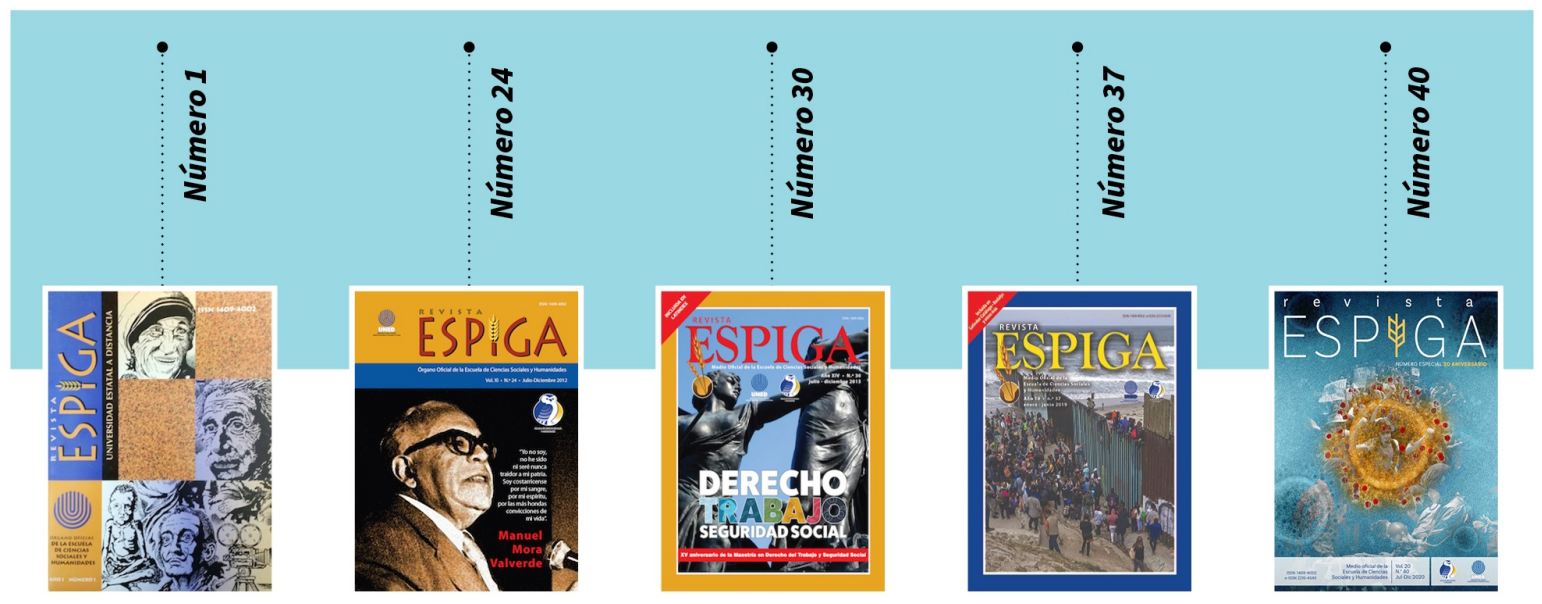

Evolución gráfica de la portada de la revista a to targo de dos décadas. A la izquierda, la número 1; a la derecha, la número 40 . Se aprecia, también, el cambio en el imagotipo (representación gráfica de una marca a través de una o varias palabras junto a un icono), que mantiene la espiga en las tres realizaciones.

Uno de los libros del Antiguo Testamento, más bellamente escrito y socialmente interesante, es el libro de Rut, la espigadora en los campos de Booz ${ }^{11}$. En este y en otros pasajes de la Biblia, la espiga está asociada a un significado humanista, social y jurídico en las leyes del antiguo Israel. Asimismo, su contenido convertido en pan y alimento, en la historia y cultura del cristianismo, pasó a significar vida, energía, luz y vida después de la vida.

Por tanto, para los fundadores de la Revista, ya en contexto de la academia, Espiga significaba y significa un semillero de pensamiento crítico, de nuevas ideas, nuevas líneas de investigación, que dieran vida y realimentaran el quehacer académico de la Escuela y de la Universidad. Revista Espiga nació con el claro propósito de impulsar y apoyar la investigación, condición esencial e indispensable -como indica Clark- de la calidad y la excelencia académica en la Universidad.

Este perfil y metas de trabajo están expresados en el editorial del primer número y en la guía para los colaboradores de la Revista. Si se revisa dicha guía, se observará el trabajo serio del Consejo Editorial al establecer requisitos y estándares de calidad de nivel internacional.

De esta manera, se logró, desde el inicio, mantener pertinencia, selectividad y calidad en los trabajos publicados en las tres secciones de la Revista: a) Tema central, b) Aportes y c) Vida Universitaria. Esta última, divulga trabajos sobre la vida en la Universidad (conferencias, simposios, resultados de congresos, etc.).

\footnotetext{
10 Lester Osorno, Xinia Zúñiga Muñoz y Pedro Ramírez Acosta, «La Escuela de...», 61-62.
}

11 Rut, 1 y 2, en Biblia de Jerusalén, Ed. Desclée de Brouwer, Bilbao, 1967, 276-277. 
Uno de los mayores logros de la Revista fue motivar y alcanzar la participación de profesores de la Escuela, en líneas y proyectos de investigación, cuyos resultados eran y son publicados en Espiga. A estos esfuerzos se sumó la participación de investigadores y escritores reconocidos a nivel nacional, como Miguel Picado Gatjens de la Universidad Nacional (UNA), José Luis Vega Carballo de la UCR, Rodrigo Quesada Monge de la UNA; y, a nivel internacional, Alfonso López Quintás de la Universidad Complutense de Madrid, España, Herón Pérez Martínez de la Universidad de Michoacán, México, Carlos Rojas Ortiz de la Universidad de Puerto Rico y otros más que nos han honrado con sus escritos.

No menos importante en esta labor de espigar fue lograr la indexación de la Revista en Latindex, a partir del número 13. No es casual que ese número tuvo como tema central la filosofía y su búsqueda humanista y científica, desde los clásicos griegos hasta el presente. En el editorial de ese número escribimos la siguiente reflexión:

¿Es necesaria y útil la filosofía? Algunos se atreverían a pensar que es cosa del pasado, de los antiguos y de los medievales, pero que en la sociedad moderna y contemporánea no tiene cabida, en especial, una parte de ella, la ética y la moral. Efectivamente vivimos la euforia del avance de las ciencias y de los grandes descubrimientos científicos en el orden del espacio, de la vida, de las telecomunicaciones y de todas las áreas del conocimiento. (....) ¿Qué queda por conocer? ¡Nunca como ahora el ser humano se ha sentido más cerca de aquella frase del Génesis 3.5, «Y seréis como dioses»!

Esta frase es precisamente el título de la obra de Erick Fromm, en la que, como en Anatomía de la destructividad humana, analiza con profundidad los desvaríos de la sociedad moderna ilustrada... ${ }^{12}$

Ese y demás editoriales revelan el nivel del trabajo intelectual de la Revista. Gracias a este esfuerzo conjunto de todos los que trabajamos en Espiga nos permitió lograr la indexación de la Revista. ¿Qué es Latindex? Es una red de instituciones, del más alto nivel, que reúne y disemina información sobre publicaciones científicas producidas en España, Portugal y América Latina. Pertenecer a ella implica cumplir con ciertos estándares de calidad. Es un prestigio, pero a la vez, un compromiso, porque se puede perder la afiliación.

\section{¿Problemas en la siembra?}

Los hubo. Desde su aprobación, Espiga enfrentó el problema de financiamiento. El presupuesto otorgado cubría solamente los costos de edición e impresión por parte de la Dirección Editorial de la Euned. Por diez años el primer director de la Revista y los miembros del Consejo Editorial hicieron su trabajo «ad honorem». Sospechamos, al volver sobre estos hechos del pasado, que la idea de la importancia de una revista académica no calaba, a nivel de convencimiento, en la mentalidad administrativa de la Universidad.

\footnotetext{
12 Pedro Ramírez-Acosta, «Expansión y Calidad de los Posgrados: Estudio del Modelo de la UNED», Revista Espiga 2, n. 4 (2001): 137-56; Erick Fromm, Y seréis como dioses (Barcelona: Editorial Paidós, 1974).
} 
Otro problema relacionado con criterios de calidad, a nivel de Dirección y Consejo Editorial, fue tener que rechazar trabajos de compañeros profesores porque no cumplían con los criterios establecidos. En muchos casos sirvió para tomar conciencia que investigar y publicar los resultados es un aprendizaje necesario en la academia, que se alcanza a prueba de errores y de fuerza de voluntad para domar la chispa creativa.

Un tercer problema sucedió con la edición de un número de la Revista, en la que se filtraron errores de dicción y de traducción. Al señalarlos un distinguido compañero, me dio enorme vergüenza por mi descuido, como Director. Inmediatamente pensé y elevé mi renuncia irrevocable ante el Consejo Editorial, en el que era miembro el director de la Escuela. Mi renuncia no fue aceptada. Era el año $\mathrm{V}$ de la Revista. Hubo, sin duda, otros problemas menores, que la pasión por el trabajo académico y el deseo de servir a la universidad ayudaron a superarlos.

\section{Nuevos surcos para espigar: reflexiones finales}

Dejé la dirección de Espiga en el 2009, para acogerme a la pensión. Era el año VII de la Revista. En ese período se publicaron 19 números, más el numero 23, que me correspondió coordinar por razón de su tema central. A la distancia de once años, desde mi retiro, me complace reconocer los nuevos aires que han oxigenado el crecimiento de Revista Espiga y, que a la fecha, se han publicado 42 números, ahora también en formato digital.

Sin embargo, a esta altura de los tiempos sigo pensando, igual que ayer, en los desafíos actuales para la Revista o lo que metafóricamente podría llamarse «nuevos surcos para espigar». Desafíos que han estado y están vinculados con el crecimiento y mejoramiento de la calidad en la Escuela y en la universidad. No está de más recordar el papel de liderazgo académico que ha protagonizado la Escuela en el pasado cercano, como fue en la creación de los posgrados ${ }^{13}$, creación de nuevas carreras, la Reforma de los Estudios Generales ${ }^{14}$, la misma creación de Espiga y la activa participación en el Tercer Congreso (2006), para la propuesta de creación de la Vicerrectoría de Investigación. La moción sexta y séptima, cuyo borrador fue elaborado por la Escuela, ponía énfasis en la urgencia de dicha Vicerrectoría, en la necesidad de integrar, bajo el modelo sistémico, los diversos componentes: institutos, centros, programas o redes con diversas líneas de investigación. Asimismo, se proponía la articulación entre investigación, docencia, producción de materiales, extensión y publicación de los resultados de investigación.

En este marco de acciones y propuestas ¿Qué papel debe jugar Revista Espiga con miras al futuro? La función de una revista no debe ser solamente receptiva, esto es recibir y difundir conocimientos, sino también propositiva. Hay dos vertientes que Espiga puede revisar y profundizar. Una es a lo interno de la institución, ¿Cómo hacerlo? Planteando líneas y temas de investigación sobre la pertinencia y eficacia de las estructuras, que los mismos profesores 0 funcionarios administrativos pueden desarrollar, en líneas aprobadas de 
investigación o como tesis de graduación en los diferentes posgrados. Se puede plantear, también, preguntas irreverentes como las siguientes, que motiven la investigación y la innovación:

- ¿En la actualidad favorece la calidad y la investigación la estructura de Programas y Cátedras?

- ¿Por qué, después de 45 años de existencia de la Universidad y 30 de las escuelas, no existen en ellas institutos y centros de investigación, algo como el CINED de la Escuela de Educación, que inició labores en el 2016?

- ¿Existe en la UNED «excelencia selectiva» o lo que otros llaman «meritocracia académica», vinculadas ambas a la carrera profesional académica, esto es al talento y al esfuerzo aplicados a grados obtenidos y a número de publicaciones científicas?

- ¿Cómo y qué requisitos académicos existen para acceder a los puestos de Dirección, Encargado de Programa y de Cátedra?

- ¿La inmovilidad (o falta de alternabilidad periódica) en los puestos de Encargado de Programa y de Cátedra favorece la calidad académica?

- Los posgrados nacen y son de las escuelas, ¿Cómo retroalimentan estos a sus escuelas para elevar la calidad en la docencia y la investigación?

- En el 2016, la directora del SEP señalaba, en su plan de trabajo: «Es crucial fortalecer la interrelación entre el SEP y las escuelas académicas». ¿Se ha logrado ese fortalecimiento después de cinco años?

Una segunda vertiente surge de la relación entre investigación y la sociedad de la Cuarta Revolución Industrial o Revolución Digital. Costa Rica es un país privilegiado geopolíticamente, en vías de desarrollo, abierto al asentamiento de nuevas empresas e industrias internacionales que provocan y suscitan nuevas necesidades y cambios en el trabajo, en el sistema de empleo y en la cultura general. Las universidades deben afrontar las demandas de esta nueva sociedad industrial digital.

¿Cuáles son las características de esta nueva sociedad? Una es la velocidad cada vez más acelerada de los cambios. Desde la primera Revolución de la agricultura, hace 10000 años, a la gran revolución política, humanista científica de los griegos, pasaron 9000 años, aproximadamente. En cambio, las últimas cuatro revoluciones industriales se han llevado a cabo en menos de doscientos años.

¿Cómo enfrentar estos inusitados cambios en todos los ámbitos de la sociedad? A mediados del siglo pasado, Ortega y Gasset escribía: «Las viejas y famosas universidades inglesas preparan a sus estudiantes con una sólida formación clásica humanista para enfrentar no solo los problemas del presente, sino también los cambios en el futuro». Al respecto ¿Cuáles y cómo son los contenidos curriculares de nuestros programas de grado y posgrado? ¿Cuál es el perfil de formación que necesitamos para el desarrollo integral de la nueva

\footnotetext{
13 Pedro Ramírez-Acosta, «Expansión y Calidad...

14 José Luis Torres, «Los Estudios Generales en la Universidad Estatal a Distancia», Revista Espiga 6, n. ${ }^{\circ} 12$ (2006): 1-20.
} 
sociedad digital? ¿Estamos formando a nuestros estudiantes para enfrentar esos cambios?

Otra característica de la nueva sociedad postindustrial es la urgencia de nuevas ciencias, relacionadas con las demandas de carreras STEM (Science, Technology, Engineering and Mathematics). Es preocupante lo que señala el último Informe del Estado de la Educación: Solo el $16 \%$ de la oferta académica de las universidades en Costa Rica responde a las demandas STEM ${ }^{15}$. ¿Cuáles son algunas de estas nuevas ciencias, a cuyo estudio e investigación se deben avocar las escuelas, los centros e institutos de investigación de la Universidad? La ciencia de datos, imprescindible en la actualidad, para la toma de decisiones. Robótica e inteligencia artificial, nanotecnología, biotecnología y secuenciación genética, computación cuántica, finanzas: inversiones y banca, comunicación y negocios digitales, comercio internacional y cadenas globales de valor, humanidades con pensamiento crítico, entre otras.

No es nada fácil enfrentar estos desafíos. Revista Espiga debe proponer y ayudar a romper las barreras de comodidad y de asentamientos administrativos en la Escuela, barreras burocráticas e ideológicas, como advierte Francisco González, actual rector de la UNA, para abrir espacios de reflexión e investigación en esas nuevas áreas del conocimiento científico y humanista.

En relación con esta necesidad de romper barreras, es gratificante reconocer los avances de la UNED en el campo de la investigación. La creación y el trabajo de la Vicerrectoría de Investigación (2006) ha contribuido a generar cambios y mejoras en el desempeño académico. Datos actuales, como los siguientes: 292 investigadores y 272 estudiantes vinculados a los procesos de investigación, cuatro centros de investigación, un instituto, dos laboratorios de investigación y nueve revistas científicas, la mayoría de ellas indexadas en redes internacionales, ponen a la UNED en la ruta hacia la calidad y la excelencia académica. Trabajos de investigación como: «Aportes de Vigotsky y la pedagogía crítica para la transformación del diseño curricular en el siglo XXI», de César Toruño Arguedas (Innovaciones Educativas, n.o 33, 2020); «La Universidad del futuro y la 4.0 Revolución. Hacia una universidad innovadora. Análisis prospectivo», de Roberto Carvajal (Revista Electrónica Calidad en la Educación Superior, Vol. 11, 2, 2020) y «Estudio de los factores desencadenantes del síndrome de quemado en las cuatro escuelas y el Sistema de Posgrado de la UNED», de Randall Trejos Alvarado (Revista Electrónica Calidad en la Educación Superior, Vol. 11, 2, 2020)ํㅜ, constatan el interés y el esfuerzo de los académicos por responder a los retos de la nueva sociedad digital.

Terminamos estas reflexiones reafirmando: No hay ni habrá calidad en las carreras de grado y posgrado y en la producción de los materiales didácticos, si

${ }^{15}$ Consejo Nacional de Rectores, Informe del Estado de la Educación (San José: CONARE, 2020); Juan Diego Córdoba González, «Carreras del futuro demandan reforma en oferta de universidades», La Nación, 17 de abril de 2021, 6-7. 
no hay investigación y nuevos conocimientos. Y las investigaciones requieren de una o más revistas, que difundan y promuevan los nuevos conocimientos. Revista Espiga ha cumplido esta misión y le deseamos mayores y mejores logros en el futuro.

\section{Formato de citación según APA}

Ramírez-Acosta, P. J. (2022). Revista Espiga en la memoria histórica de la ECSH y de la UNED. Revista Espiga, 21 (43), 1-13.

Formato de citación según Chicago-Deusto

Ramírez-Acosta, Pedro J. «Revista Espiga en la memoria histórica de la ECSH y de la UNED». Revista Espiga 21, n. 43 (enero-junio, 2022): 1-13

\footnotetext{
${ }^{16}$ Randall Trejos Alvarado, «Estudio de los factores desencadenantes del síndrome de quemado en las cuatro Escuelas y el Sistema de Posgrado de la UNED», Revista Electrónica Calidad en la Educación Superior 11, n. - 7 (2020): 214-260. La investigación del profesor Trejos Alvarado es novedosa e interesante, por cuanto se acerca, con cierto rigor científico, al problema de la calidad del trabajo del «profesor quemado», conocido como «síndrome burnout», en programas, cátedras y posgrados. ¿No debió preguntarse, también, por la inmovilidad en el cargo y requisitos académicos para acceder a él de parte del «profesor quemado», como un factor esencial de la calidad académica?
} 


\section{Referencias}

Barrantes Echavarría, Rodrigo. Educación a Distancia. San José: EUNED, 1998.

Barrow, Clyde W. «La estrategia de la excelencia selectiva». Perfiles educativos 19, n. 77 (1997): 76-77.

Clark, Burton. The Higher Education System: Academic Organization in Cross National-Perspectives. Berkeley: University of California Press, 1983.

Consejo Nacional de Rectores. Informe del Estado de la Educación. San José: CONARE, 2020.

Córdoba González, Juan Diego. «Carreras del futuro demandan reforma en oferta de universidades». La Nación, 17 de abril de 2021. 6-7.

Osorno, Lester, Xinia Zúñiga Muñoz y Pedro Ramírez Acosta. «La Escuela de Ciencias Sociales y Humanidades en el treinta aniversario de la UNED». Revista Espiga 8, n. 18 (2009): 61-76. Doi: https://doi.org/10.22458/ re.v8i18.1044.

Ramírez Acosta, Pedro. «Elementos para el estudio de viabilidad de un Sistema de Educación a Distancia para la Universidad Autónoma de Nicaragua». Tesis de Maestría Académica, Universidad de Costa Rica. San José, Costa Rica, 1984.

Ramírez Acosta, Pedro. «Expansión y calidad de los Posgrados: Estudio del modelo de la UNED». Revista Espiga 2, n. ${ }^{\circ} 4$ (2001): 137-156.

Torres, José Luis. «Los Estudios Generales en la Universidad Estatal a Distancia». Revista Espiga 6, n. 12 (2006): 1-20.

Trejos Alvarado, Randall. «Estudio de los factores desencadenantes del síndrome de quemado en las cuatro Escuelas y el Sistema de Posgrado de la UNED». Revista Electrónica Calidad en la Educación Superior 11, n. ${ }^{\circ} 7$ (2020): 214-260.

Tünnermann, Carlos. La Educación Superior frente al cambio. San José: EDUCA/ CSUCA, 1997.

UNED. Acuerdos sobre la creación de la Dirección de Docencia. Edición provisional, 1990.

Unesco. «Declaración Mundial sobre la Educación Superior». DIALOGO, México OPI-LAC-Unesco, 1998. 